\title{
Cutibacterium acnes Induces the Expression of Immunosuppressive Genes in Macrophages and is Associated with an Increase of Regulatory T-Cells in Prostate Cancer
}

\author{
(1) Sabina Davidsson, ${ }^{a}$ Jessica Carlsson, ${ }^{a}$ Larry Greenberg, ${ }^{\mathrm{b}}$ Jonny Wijkander, ${ }^{\mathrm{c}}(1)$ Bo Söderquist, ${ }^{\mathrm{d}, \mathrm{e}}(\mathbb{C})$ Ann Erlandsson ${ }^{\mathrm{a}, \mathrm{b}}$ \\ aDepartment of Urology, Faculty of Medicine and Health, Örebro University, Örebro, Sweden \\ bDepartment of Environmental and Life Sciences/Biology, Faculty of Health, Science and Technology, Karlstad University, Karlstad, Sweden \\ cDepartment of Health Sciences, Faculty of Health, Science and Technology, Karlstad University, Karlstad, Sweden \\ dSchool of Medical Sciences, Faculty of Medicine and Health, Örebro University, Örebro, Sweden \\ eDepartment of Laboratory Medicine, Faculty of Medicine and Health, Örebro University, Örebro, Sweden
}

\begin{abstract}
Tumors and infectious agents both benefit from an immunosuppressive environment. Cutibacterium acnes (C. acnes) is a bacterium in the normal skin microbiota, which has the ability to survive intracellularly in macrophages and is significantly more common in prostate cancer tissue compared with normal prostate tissue. This study investigated if prostate cancer tissue culture positive for $C$. acnes has a higher infiltration of regulatory T-cells (Tregs) and if macrophages stimulated with $C$. acnes induced the expression of immunosuppressive genes that could be linked to an increase of Tregs in prostate cancer. Real-time PCR and enzyme-linked immunosorbent spot assay (ELISA) were used to examine the expression of immunosuppressive genes in human macrophages stimulated in vitro with $C$. acnes, and associations between the presence of $C$. acnes and infiltration of Tregs were investigated by statistically analyzing data generated in two previous studies. The in vitro results demonstrated that macrophages stimulated with $C$. acnes significantly increased their expression of PD-L1, CCL17, and CCL18 mRNA and protein $(p<0.05)$. In the cohort, Tregs in tumor stroma and tumor epithelia were positively associated with the presence of $C$. acnes $(P=0.0004$ and $P=0.046$, respectively). Since the macrophages stimulated with $C$. acnes in vitro increased the expression of immunosuppressive genes, and prostate cancer patients with prostatic $C$. acnes infection had higher infiltration of Tregs than their noninfected counterparts, we suggest that $C$. acnes may contribute to an immunosuppressive tumor environment that is vital for prostate cancer progression.

IMPORTANCE In an immune suppressive tumor microenvironment constituted by immunosuppressive cells and immunosuppressive mediators, tumors may improve their ability to give rise to a clinically relevant cancer. In the present study, we found that $C$. acnes might contribute to an immunosuppressive environment by recruiting Tregs and by increasing the expression of immunosuppressive mediators such as PD-L1, CCL17, and CCL18. We believe that our data add support to the hypothesis of a contributing role of $C$. acnes in prostate cancer development. If established that $C$. acnes stimulates prostate cancer progression it may open up avenues for targeted prostate cancer treatment.
\end{abstract}

KEYWORDS Cutibacterium acnes, macrophages, prostate cancer, regulatory T-cells, cancer, prostate

mmune escape, defined by the incapacity of the immune system to eliminate tumor cells and thereby prevent clinically relevant cancer, is one of the hallmarks of cancer $(1,2)$. To avoid being eliminated, tumors can create an environment comprised of immune cells and immune-related proteins with immunosuppressive functions. The supportive mechanisms for an immunosuppressive environment are multifactorial, but one potentially strongly
Editor Jan Claesen, Lerner Research Institute Copyright $\odot 2021$ Davidsson et al. This is an open-access article distributed under the terms of the Creative Commons Attribution 4.0 International license.

Address correspondence to Ann Erlandsson, ann.erlandssson@kau.se.

The authors declare no conflict of interest.

Received 22 September 2021

Accepted 30 November 2021

Published 22 December 2021 
contributing factor is related to infectious agents that benefit from a dampened immune response in a similar manner to tumors.

Cutibacterium acnes (C. acnes) is a bacterium that can survive intracellularly in macrophages, enabling it to evade the host immune response (3). Several factors contribute to this intracellular persistence, such as catalase production and a thick tightly cross-linked cell wall (4). However, C. acnes also produces arginine deiminase (5), which can reduce host T-cell function (6). In line with this, it has been demonstrated that monocytes treated with interleukin (IL)-4, granulocyte-macrophage colony-stimulating factor (GM-CSF), and C. acnes induce Tcell differentiation to immunosuppressive regulatory T-cells (Tregs) (7). C. acnes can induce the expression of both pro- and anti-inflammatory cytokines (8), but despite the possible link between $C$. acnes and increase of Tregs, few studies have focused on the antiinflammatory effects of $C$. acnes. However, it has been shown that $C$. acnes induces IL-10 expression in adherent peripheral blood mononuclear cells; that is, monocytes (9).

C. acnes normally constitutes part of the microbiota on human skin and mucous membranes but can cause insidious long-lasting chronic infections at various sites including the prostate $(3,10-12)$. It has also been identified as the predominant microorganism in prostatic tissue specimens obtained from prostate cancer patients $(8,13,14)$. C. acnes has been classified into different subtypes (IA, IB, IC, II, and III), and genotyping has revealed that all subtypes are capable of colonizing the prostate although type II has been reported as the most prevalent subtype $(8,11,14)$.

The most abundant immune cell in the tumor environment is the macrophage, which has the ability to polarize into a tumor-inhibiting M1 phenotype or an M2 phenotype with tumor-promoting and immunosuppressive functions $(15,16)$. A number of different immuno-mediators, such as programmed death-ligand 1 (PD-L1), macrophage colony stimulation factor (M-CSF), C-C motif chemokine ligand 2 (CCL2), C-C motif chemokine ligand 17 (CCL17), C-C motif chemokine ligand 18 (CCL18) and C-C motif chemokine ligand 22 (CCL22), predominantly released by $M 2$ macrophages, have been linked to increased numbers of both macrophages and Tregs in tumor tissue (15, 17-19). An immune-suppressive tumor environment constituted by Tregs and $\mathrm{M} 2$ macrophages along with immunosuppressive mediators such as CCL17, CCL18, CCL22, and PD-L1 can assist tumor development and progression $(20,21)$. In prostate cancer, higher infiltration of Tregs and M2 macrophages in tumor tissue has been associated with worse prognosis (1, 2, 22-25).

The aim of the present study was to investigate if higher levels of Tregs in prostate cancer tissue is associated with the presence of $C$. acnes and whether macrophages stimulated with $C$. acnes increase their expression of immunosuppressive genes that contribute to recruitment of Tregs in prostate cancer.

\section{RESULTS}

C. acnes stimulated macrophages. To investigate the impact of $C$. acnes on secretion of immunosuppressive mediators from macrophages, we stimulated macrophages obtained from healthy male donors with $C$. acnes strains isolated from prostate cancer tissue. We selected a macrophage and $C$. acnes ratio of 10 multiplicity of infection (MOI) based on previous experiment incubating $C$. acnes with cultured prostate cancer cells (14). After a 48-h incubation of $\mathrm{MO}$ macrophages with $10 \mathrm{MOI}$ C. acnes of type IA or II, the macrophages had transformed from an elongated shape to a rounder shape with a foamy appearance. At this point, C. acnes of both type IA or II, had increased in number (Fig. 1A-D).

mRNA and protein expression in macrophages stimulated with $C$. acnes. Stimulation of macrophages with $C$. acnes type IA or type II produced a significantly increased mRNA expression of CCL17 (type IA: $P=0.006$; type II: $P=0.029$ ) and CCL18 (type IA: $P=0.002$, type II: $P=0.023)$. Stimulation with type IA also produced a significant increase in PD-L1 $(P=0.032)$. The effects of the stimulation on the mRNA expression level are presented in Fig. $2 A$ as log $\Delta C t$ values. The fold change increases in the mRNA expression in the stimulated macrophages compared with their unstimulated counterparts were 100-660 for CCL17, 190-790 for CCL18, and 2-40 for PD-L1. No significant differences were seen in expression of the mRNA encoding M-CSF, CCL2, and CCL22 $(P>0.05)$ (Supplement 1 in the supplemental material). There were also no 
A

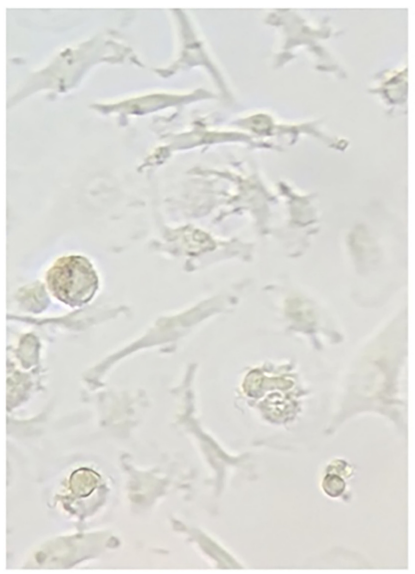

B

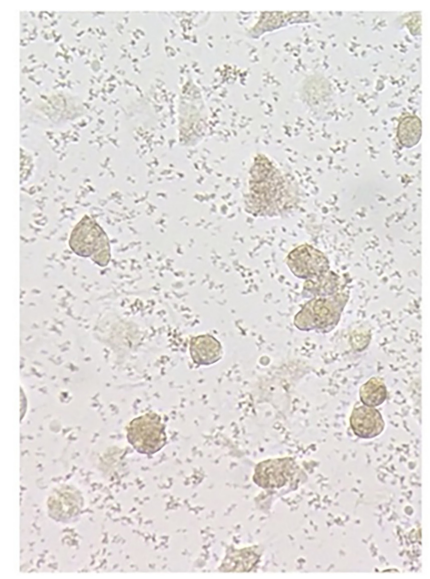

C

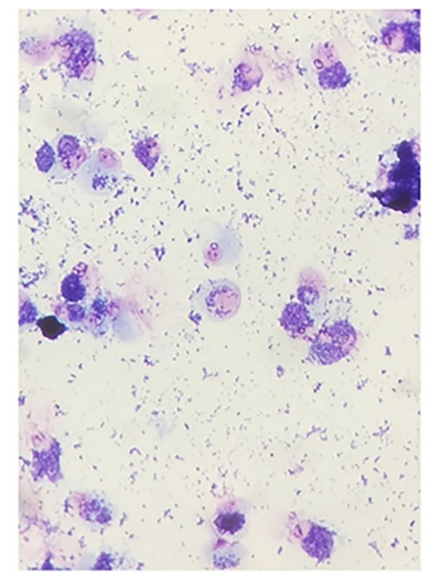

D

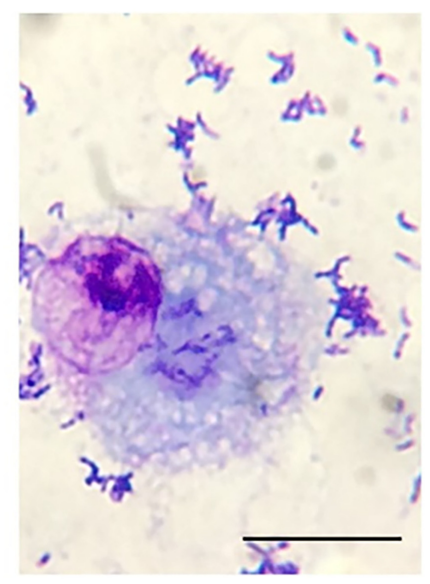

FIG 1 Representative images of the change of macrophage morphology after stimulation with C. acnes type II. (A) Unstimulated macrophages in culture plate after $48 \mathrm{~h}$ of incubation (x20). (B) Macrophages in culture plate stimulated with $10 \mathrm{MOI}$ C. acnes type II for $48 \mathrm{~h}$ of culture (x20). (C) Macrophages stimulated with $10 \mathrm{MOI}$ C. acnes type II for $48 \mathrm{~h}$ of culture followed by Giemsa staining (x20). (D) Macrophages stimulated with 10 MOI C. acnes type II for $48 \mathrm{~h}$ of culture, scraped off the culture plate and stained with Giemsa staining $(\times 100$, scale bar $30 \mu \mathrm{m})$.

significant difference in expression of any of the mRNA between the macrophages stimulated with $C$. acnes type IA and those stimulated with type II.

For the genes that demonstrated a significant increased expression of mRNA (i.e., PD-L1, CCL17, and CCL18), enzyme-linked immunosorbent spot assays (ELISAs) were performed to confirm significant changes in protein levels in the culture media. Macrophages stimulated with $C$. acnes type IA or type II demonstrated increased levels of all three proteins: CCL17 between 97-289 pg/ml (type IA: $P=0.001$; type II: $P=0.009$ ), CCL18 between 560-9148 pg/ml (type IA: $P=0.022$; type II: $P=0.041$ ), and PD-L1 between 28-147 pg/ml (type IA: $P=0.002$; type II: $P=0.009$ ) proteins (Fig. $2 \mathrm{~B}$ ). There were no significant differences in protein levels between the macrophages stimulated with $C$. acnes type IA and those stimulated with type II $(P>0.05)$.

Associations between infiltration of Tregs and $C$. acnes in prostate tissue from patients with prostate cancer. Associations between the presence of $C$. acnes and infiltration of Tregs were investigated by statistically analyzing data generated in two previous

A

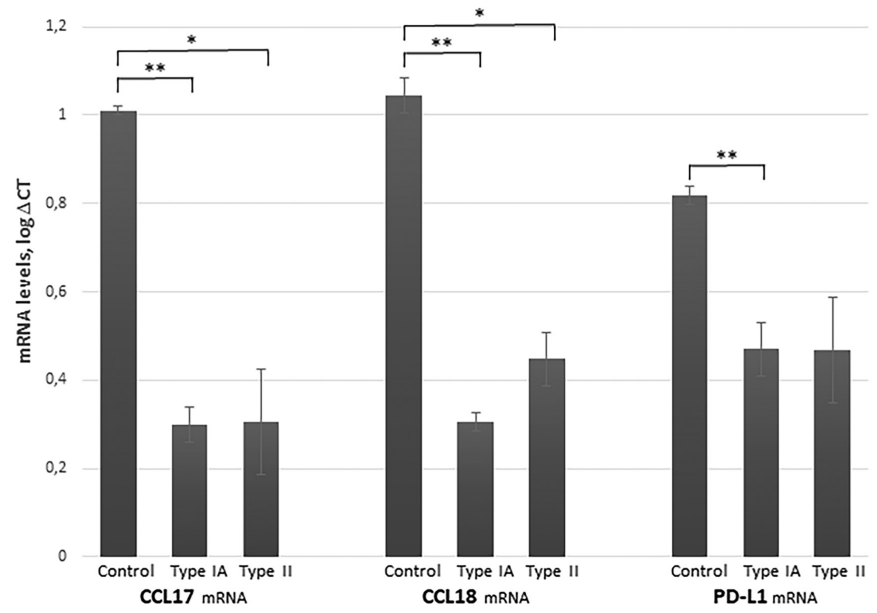

B

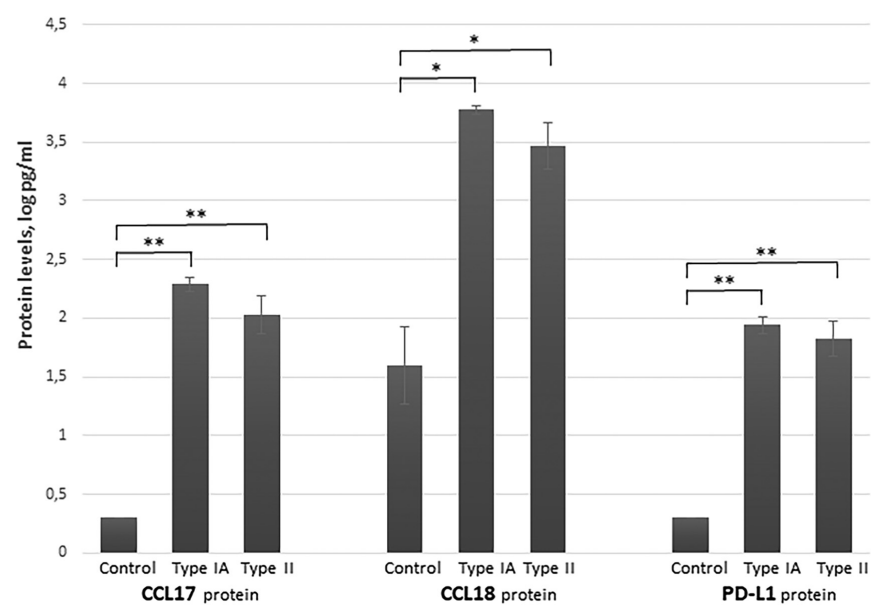

FIG 2 Expression of CCL17, CCL18, and PD-L1 in unstimulated macrophages (control) and macrophages stimulated with C. acnes type IA or type II. (A) mRNA expression in unstimulated control and C. acnes type IA or type II stimulated macrophages, presented as mean $+/$ - SD of log $\Delta C T$ (i.e., the mRNA expression of the gene of interest minus the expression of the housekeeping gene POLR2F). The lower the $\Delta \mathrm{Ct}$ value, the higher the expression of the mRNA of interest. (B) Protein expression in unstimulated control and C. acnes type IA or type II stimulated macrophages, presented as mean +/- SD of log pg/ml. The statistical analysis were performed using repeated measurements ANOVA with two within subject factors (treatment and replicates). The treatments were unstimulated (control), stimulated with $C$. acnes type IA or with $C$. acnes type II. The number of replicates were three, done with three different macrophage donors. ${ }^{*} P<0.05$, ${ }^{* *} P<0.01$. 
TABLE 1 Infiltration of Tregs in 151 tumors (stromal and epithelial compartment) from 137 prostate cancer patients with and without prostatic $C$. acnes infection

\begin{tabular}{lcc}
\hline Infiltration of Tregs & Patients with no C. acnes & Patients with C. acnes \\
\hline Tregs in tumor stroma (\%) & $17(30)$ & $39(70)$ \\
$\quad$ Yes & $57(60)$ & $38(40)$ \\
No & & \\
Tregs in tumor epithelia (\%) & $15(33)$ & $30(67)$ \\
Yes & $59(56)$ & $47(44)$ \\
No &
\end{tabular}

studies investigating the presence of $C$. acnes (14) and Tregs (24), respectively, in the same cohort of patients. Prostate cancer is generally multifocal with tumors presenting histological differences. In the present study, we analyzed the association between the presence of C. acnes and Tregs in 151 tumors of the prostate gland obtained from 137 prostate cancer patients. Among these 137 men, 73 cases were $C$. acnes positive and 64 cases were $C$. acnes negative, the 14 additional tumors from these 137 men were from $10 \mathrm{C}$. acnes negative and $4 \mathrm{C}$. acnes positive cases with multiple tumors. The new statistical analysis performed in the present study demonstrated that Tregs, both in the tumor stromal and the tumor epithelial compartment, were positively associated with the presence of $C$. acnes ( $P=0.0004$ and $P=0.046$, respectively) (Table 1$)$.

\section{DISCUSSION}

An increasing number of studies have demonstrated that chronic infections are often associated with increased infiltration of anti-inflammatory cells, and that this infiltration can support cancer initiation and progression $(8,26,27)$. In the present study, we investigated whether $C$. acnes was able to induce the expression of immunosuppressive proteins in macrophages, which are among the most abundant immune cells in tumor environments. Our results showed that macrophages cultured with $C$. acnes increased their expression of three proteins that are involved in recruitment and accumulation of Tregs in vivo. In addition, we found that the number of Tregs was significantly higher in prostate cancer tissue that was culture-positive for $C$. acnes compared with nonculture-positive tissue.

M2 macrophages and Tregs are immunosuppressive cells that under normal conditions prevent autoimmune reactions and excessive inflammation. The side effect of this immunosuppression is the loss of both anti-tumor immunity and effective elimination of chronic bacterial, viral, or fungal infections $(15,28-30)$. In the present study, we investigated the expression of mRNA encoding M-CSF, CCL2, CCL17, CCL18, CCL22, and PD-L1 in macrophages stimulated with $C$. acnes. The main function of M-CSF, CCL2, and CCL22 is to recruit M2 macrophages and/or Tregs. In our study, the expression of these genes in the macrophages was already high at a basal level, and no significant increase could be determined (Supplement 1). This may have been due to the pretreatment of monocytes with M-CSF that we performed in vitro for the differentiation of monocytes into macrophages. However, a significantly increased expression of both mRNA and the corresponding proteins regarding CCL17, CCL18, and PDL1 was observed in macrophages stimulated with C. acnes. These genes, CCL17, CCL18, and $P D-L 1$, are mainly expressed in $M 2$ macrophages, indicating that $C$. acnes is able to polarize the macrophage into an immunosuppressive $M 2$ phenotype with the ability to recruit Tregs (15). In line with our results, a recently published study on gastric cancer identified $C$. acnes as a possible cause of increased polarization of macrophages against an M2 phenotype and increased cancer progression (31). Our microscopic examination of the macrophages stimulated with $C$. acnes revealed a rounder shape with a foamy appearance, as previously demonstrated in M2 macrophages with PD-L1 expression (32). We did not detect any significant differences in expression of the genes between macrophages stimulated with $C$. acnes type IA and those stimulated with type II. However, other studies have found differences in the ability of $C$. acnes types to stimulate expression of immune-related genes (9). One study demonstrated a higher IL-10 expression in monocytes after stimulation with C. acnes type II compared 
with type I, though the induction of an interferon- $\gamma$ response appeared to be similar between types I and II (9).

C. acnes is a Gram-positive, facultative anaerobic bacterium that constitutes part of the normal microbiota on human skin and mucosal membranes. Nevertheless, this opportunistic bacterium can cause both acute and low-grade chronic infections of greater or lesser severity (10-12). The main reason why $C$. acnes is able to evade the host immune response is its capacity to survive and proliferate intracellularly in macrophages, and it is worth noting that $C$. acnes has been suggested as an etiology of the granulomatous disorder seen in sarcoidosis $(3,12,13,33)$. One factor that can contribute to the intracellular survival of $C$. acnes is the production of catalase (4). In addition to catalase production, $C$. acnes has a cell wall with high resistance to degrading and oxidizing enzymes; and it also produces arginine dihydrolase (5), which contributes to depletion of arginine and subsequent reduced host T-cell function and proliferation as well as reduced development of an immunological memory (6). In line with this, it has been demonstrated that monocytes treated with IL-4, GM-CSF, and C. acnes induce a differentiation of T-cells into anti-inflammatory Tregs (7). However, it has been shown that $C$. acnes is able to induce the expression of both pro- and anti- inflammatory cytokines, with the pro-inflammatory reactions as a possible cause of DNA damage and initiation of tumorigenesis (8). IL-10 is an immunosuppressive interleukin that among other functions can stimulate differentiation of T-cells into Tregs (34). As already mentioned, it has been demonstrated that $C$. acnes can induce IL-10 expression in peripheral blood mononuclear cells, providing an opportunity for immune escape (9).

Mycobacterium tuberculosis (M. tuberculosis) is another example of a bacteria able to proliferate within macrophages. The main histological finding in tuberculosis, as for sarcoidosis, is granuloma with macrophages as the main cell type. In line with our results, macrophages stimulated with M. tuberculosis have increased PD-L1 and CCL18 expression (32, 35). Overexpression of PD-L1 correlates with worse prognosis in many cancers, including prostate cancer, and in some cancers there is an increasing use of therapeutic interventions that, for example, block PD-1 with pembrolizumab or PD-L1 with atezolizumab in order to reduce immunosuppression in the tumor environment (36).

High expression of CCL17 has been linked to worse prognosis in prostate cancer, renal cancer, and testicular cancer $(18,37,38)$, and increased expression has also been observed in chronic viral infections such as hepatitis $C$ and human papillomavirus, which are associated with malignant transformation $(39,40)$. High levels of CCL18 can be linked to cancer progression in colon cancer, breast cancer, and prostate cancer, where CCL18 not only activates recruitment of Tregs but also stimulates cancer cell migration and angiogenesis (17, 19, 41, 42). Increased expression has also been seen in gastric biopsies chronically infected with Helicobacter pylori and in nasopharyngeal carcinoma tissue with a latent infection of EpsteinBarr virus $(43,44)$.

The purpose of this study was to investigate whether $C$. acnes can induce the expression of immunosuppressive genes in macrophages, and whether there is an association between C. acnes and Tregs in prostate cancer. The study does have some important limitations. Only nine in vitro experiments were performed, using macrophages from three different blood donors, and the gene expression was examined using one specific ratio of 1:10 (10 MOI) of macrophage and live $C$. acnes at one time point. The most significant strengths are that the in vitro experiments were performed with $C$. acnes strains isolated from prostate cancer tissue and macrophages isolated from male blood donors, and the relatively large number of men included in the investigation of associations between Tregs and C. acnes infiltration.

This study found that macrophages stimulated with C. acnes increased the expression of genes involved in recruiting immunosuppressive Tregs. It also demonstrated significantly higher levels of Tregs in prostate cancer tissue with growth of $C$. acnes compared with culturenegative tissue. We suggest that $C$. acnes may contribute to an immunosuppressive status that facilitates a tumor-stimulating environment, which is vital for prostate cancer progression, and highlight the importance of further investigation regarding the role of infectious agents and other immune modifying factors in prostate cancer development and progression. In 
future studies, it is of great interest to investigate presence of $C$. acnes and expression of immunosuppressive proteins in normal prostate tissue and evaluate differences between men who develops prostate cancer compared with those without a prostate cancer diagnosis.

\section{MATERIALS AND METHODS}

Isolation of human monocytes and differentiation to MO macrophages. Buffy coats from healthy anonymous male blood donors were obtained from the Division of Clinical Immunology and Transfusion Medicine, Uppsala University Hospital, Uppsala, Sweden. Monocytes were isolated from the buffy coats by gradient centrifugation using Ficoll-Paque PLUS (GE Healthcare, Little Chalfont, UK). In short, about $50 \mathrm{ml}$ of buffy coat was diluted with an equal volume of phosphate-buffered saline $\mathrm{pH} 7.4$ containing $3 \mathrm{mM}$ Ethylene diamine tetra acetic acid (PBS/EDTA), loaded on Ficoll-Paque PLUS, and centrifuged at $900 \mathrm{~g}$ for $30 \mathrm{~min}$ at $20^{\circ} \mathrm{C}$. The separated mononuclear fraction was collected and diluted with PBS/EDTA followed by centrifugation at $500 \mathrm{~g}$ for $10 \mathrm{~min}$. Cells were suspended in PBS/EDTA and washed four times with PBS/EDTA by repeated centrifugations at $200 \mathrm{~g}$ for $10 \mathrm{~min}$. After washing, the cells were suspended in $100 \mathrm{ml} \mathrm{RPMl} 1640$ with $1.5 \mathrm{mM} \mathrm{L-gluta-}$ mine and penicillin/streptomycin solution (PEST) $(100 \mathrm{U} / \mathrm{ml}$ of penicillin and $100 \mu \mathrm{g} / \mathrm{ml}$ of streptomycin) (all from Life Technologies Carlsbad, CA). Thereafter, $2 \mathrm{ml}$ of cell suspension was seeded onto 6-well cell culture plates (Greiner Bio-One, Frickenhausen, Germany) and allowed to adhere for $1.5 \mathrm{~h}$. Nonadherent cells were removed by three washes with PBS, after which fresh RPMI 1640 supplemented with $5 \%$ fetal calf serum (FCS) and $1.5 \mathrm{mM}$ L-glutamine and PEST was added. Macrophages of M0 phenotype (approximately $2 \times 10^{6} /$ well) were obtained by culturing monocytes for 7 days in RPMI 1640, 20\% FCS, 1.5 mM L-glutamine, PEST, and $20 \mathrm{ng} / \mathrm{ml}$ macrophage colony-stimulating factor (M-CSF) (R\&D Systems, Minneapolis, MN), with media and MCSF renewal at day 3 .

Culture and quantification of $\boldsymbol{C}$. acnes. $C$. acnes types IA and II, previously isolated from clinical prostate cancer tissue (14), were subcultured in an anaerobic atmosphere $\left(80 \% \mathrm{~N}_{2}, 10 \% \mathrm{CO}_{2}, 10 \% \mathrm{H}_{2}\right)$ at $37^{\circ} \mathrm{C}$ on Fastidious Anaerobe Agar plates (4.6\% LAB 90 Fastidious Anaerobe Agar, LAB M, Lancashire, United Kingdom) supplemented with $5 \%$ horse blood. The cultured bacteria were harvested from the plate, suspended in RPMI 1640 supplemented with 5\% FCS and $1.5 \mathrm{mM}$ L-glutamine, and quantified by Bürker counting.

Stimulation of MO macrophages with C. acnes types IA and II. M0 macrophages were cultured in RPMI 1640 medium supplemented with 5\% FCS and $1.5 \mathrm{mM}$ L-glutamine, stimulated with 10 multiplicity of infection (MOI) of C. acnes type IA or type II (suspended in RPMI 1640 with 5\% FCS and $1.5 \mathrm{mM} \mathrm{L-glutamine),}$ and cultured for $48 \mathrm{~h}$. A total of nine experiments were performed using macrophages from three different blood donors. Each experiment included an unstimulated M0 macrophage (control), M0 macrophages stimulated with $10 \mathrm{MOI}$. acnes type IA, and M0 macrophages stimulated with $10 \mathrm{MOI}$. acnes type II. After $48 \mathrm{~h}$ of incubation the culture media were removed, filtered through a $0.22 \mu \mathrm{m}$ filter, and stored at $-20^{\circ} \mathrm{C}$ until being analyzed with an ELISA. The macrophages were washed with PBS before the RNA was extracted.

RNA extraction, CDNA synthesis, and quantitative PCR. The macrophages were lysed directly in the wells of the culture plates with RLT Plus Buffer (Qiagen, Hilden, Germany) and 2-mercaptoethanol (Sigma-Aldrich, Stockholm, Sweden) followed by the extraction procedure described in the RNeasy Plus Mini Kits (Qiagen, Hilden, Germany), eluted in $30 \mu \mathrm{l}$ water and stored at $-20^{\circ} \mathrm{C}$ until use. The concentration and quality of RNA in each sample was determined with spectrophotometry (Infinite M200 PRO, Tecan Trading AG, Männedorf, Switzerland), and $200 \mathrm{ng}$ extracted RNA was used for cDNA synthesis using $\mathrm{RT}^{2}$ First Strand Kits (Qiagen, Hilden, Germany). The CDNA was diluted 1:5 and stored at $-20^{\circ} \mathrm{C}$. For the gene expression analysis, primers with specificity for mRNA coding for PD-L1, M-CSF, CCL2, CCL17, CCL18, and CCL22 were used (all primer pairs were validated QuantiTect primers from Qiagen Hilden, Germany) together with $\mathrm{RT}^{2}$ SYBR green ROX qPCR Master Mix (Qiagen, Hilden, Germany). Quantitative real-time PCR (qPCR) was performed with a StepOnePlus real-time PCR system (Applied Biosystems, ThermoFisher Scientific, USA). The qPCRs were performed in a total volume of $13 \mu \mathrm{l}$ with $4 \mu \mathrm{l}$ cDNA diluted 1:5 and $200 \mathrm{nM}$ forward and reverse primers. Samples were analyzed in duplicate or triplicate if needed due to high variation. Each run included negative controls. $C$. acnes types IA and II cDNA were included in the GPCR with all primer pairs to confirm absence of genes with sequence similarity. The $\mathrm{GPCR}$ protocol consisted of initial denaturation at $95^{\circ} \mathrm{C}$ for $10 \mathrm{~min}$, followed by a two-step cycling protocol $\left(95^{\circ} \mathrm{C}\right.$ for $15 \mathrm{~s}+60^{\circ} \mathrm{C}$ for $60 \mathrm{~s}$ ) for 40 cycles. Melting curve analyses were used to determine the identity and specificity of the PCR products. Cycle threshold was recorded for each sample, and target mRNA levels were normalized using POLR2F as reference gene (validated by Qiagen Hilden, Germany). To calculate "fold change" (i.e., the differences in gene expression level between the $C$. acnes stimulated macrophage in comparison to the unstimulated macrophage), the $2^{-\Delta \Delta C T}$ method was used (45).

ELISA. Levels of CCL17, CCL18, and PD-L1 protein in the conditioned culture media from untreated M0 macrophage controls and $C$. acnes stimulated M0 macrophages were analyzed using pre-coated Quantikine ELISA kits (R\&D Systems, Inc., Minneapolis, MN) as specified by the manufacturer. For the Quantikine ELISA human CCL17/TARC immunoassay, the samples were analyzed undiluted with a standard ranging from 31 to $2000 \mathrm{pg} / \mathrm{ml}$. For the Quantikine ELISA human CCL18/PARC immunoassay, the samples were diluted 1:10 and analyzed with a standard ranging from 18.8 to $1200 \mathrm{pg} / \mathrm{ml}$. For the Quantikine ELISA human/cynomolgus monkey PD-L1/B7-H1 immunoassay, the samples were analyzed undiluted at double volume using a standard ranging from 25 to $1,200 \mathrm{pg} / \mathrm{ml}$.

Prostate cancer tissue, $C$. acnes culture confirmation, and quantification of CD4/FOXP3 + Tregs. In the present study, we investigated the association between the presence of $C$. acnes and infiltration of Tregs in 151 tumors obtained from 137 patients. These 137 men with prostate cancer was previously a part in a cohort of patients used by our group to investigate the presence of $C$. acnes in the prostate gland of men with and without prostate cancer (14). Among the 137 cases selected for our study, 100 
cases were diagnosed with prostate cancer prior to the inclusion and 37 cases were initially categorized as controls but diagnosed with prostate cancer at the following pathological anatomical examination. The 14 additional tumors were from cases with multiple tumors. By using prostate cancer tissue from this cohort we have previously also evaluated association between infiltration of Tregs and clinical outcomes in prostate cancer (24). The Treg cell positivity were determined by CD4+FOXP3+ immunohistochemical staining. An area of $10 \mathrm{~mm}^{2}$ in sections of each tumor were visually inspected for presence of Tregs in both epithelia and tumor stromal area (24). By using data from these two investigations $(14,24)$, we now assessed the relationship between $C$. acnes and Tregs.

Statistical methods. Differences in mRNA expression and protein levels between $C$. acnes stimulated and unstimulated macrophages were investigated. For calculation of mRNA expression, the $\Delta \mathrm{Ct}$ values generated by the qPCR (i.e., the mRNA expression of the mRNA of interest minus the mRNA expression of the housekeeping gene POLR2F) were used. For the calculation of the change in protein level the $\mathrm{pg} / \mathrm{ml}$ value generated by the ELISA was used. The mRNA and protein analyses were performed using repeated measurements ANOVA with two within subject factors, macrophage treatment and replicate. The different macrophage treatments were unstimulated (control), stimulated with $C$. acnes type IA or with $C$. acnes type II. The number of replicates from each of the three macrophage donors were three. Data were $\log _{10}$-transformed after testing for normality and homogeneity of variances using the Kolmogorov-Smirnov test and Levene's test for equality of variances.

Chi-square tests were used to evaluate the relationship between the presence of $C$. acnes (culture positive or culture negative) and infiltration of Tregs (present or not present) in tumor epithelia and stromal area, respectively. Among the 137 cases included in our study, 73 cases were identified as C. acnes positive and 64 cases were identified as C. acnes negative. An additional 14 tumors from cases with multiple tumors were included, among theses, 10 were $C$. acnes negative and 4 were $C$. acnes positive. All analyses were conducted in versions 22 or 24 of SPSS (IBM, Armonk, NY) and version 14 of STATA (Stata Corp., College Station, TX).

\section{SUPPLEMENTAL MATERIAL}

Supplemental material is available online only.

SUPPLEMENTAL FILE 1, PDF file, 0.7 MB.

\section{ACKNOWLEDGMENTS}

This study was supported by the Maud and Birger Gustavsson Foundation; the Lions Cancer Foundation, Sweden; the Örebro County Council Research Committee; and the Foundation for Medical Research at Örebro University Hospital, Sweden.

\section{REFERENCES}

1. De Marzo AM, Platz EA, Sutcliffe S, Xu J, Grönberg H, Drake CG, Nakai Y, Isaacs WB, Nelson WG. 2007. Inflammation in prostate carcinogenesis. Nat Rev Cancer 7:256-269. https://doi.org/10.1038/nrc2090.

2. Strasner A, Karin M. 2015. Immune infiltration and prostate cancer. Front Oncol 5:128. https://doi.org/10.3389/fonc.2015.00128.

3. Fischer N, Mak TN, Shinohara DB, Sfanos KS, Meyer TF, Brüggemann H. 2013. Deciphering the intracellular fate of Propionibacterium acnes in macrophages. Biomed Res Int 2013:603046. https://doi.org/10.1155/2013/603046.

4. Yamamoto K, Uchida K, Furukawa A, Tamura T, Ishige $Y$, Negi M, Kobayashi D, Ito T, Kakegawa T, Hebisawa A, Awano N, Takemura T, Amano T, Akashi T, Eishi Y. 2019. Catalase expression of Propionibacterium acnes may contribute to intracellular persistence of the bacterium in sinus macrophages of lymph nodes affected by sarcoidosis. Immunol Res 67:182-193. https://doi.org/10 .1007/s12026-019-09077-9.

5. Yorozu P, Furukawa A, Uchida K, Akashi T, Kakegawa T, Ogawa T, Minami J, Suzuki Y, Awano N, Furusawa H, Miyazaki Y, Inase N, Eishi Y. 2015. Propionibacterium acnes catalase induces increased Th1 immune response in sarcoidosis patients. Respir Invest 53:161-169. https://doi.org/10.1016/j.resinv.2015.02.005.

6. Popovic PJ, Zeh HJ, 3rd, Ochoa JB. 2007. Arginine and immunity. J Nutr 137:1681S-1686S. https://doi.org/10.1093/jn/137.6.1681S.

7. Kopitar AN, Ihan Hren N, Ihan A. 2006. Commensal oral bacteria antigens prime human dendritic cells to induce Th1, Th2 or Treg differentiation. Oral Microbiol Immunol 21:1-5. https://doi.org/10.1111/j.1399-302X.2005.00237.x.

8. Brüggemann H, Al-Zeer MA. 2020. Bacterial signatures and their inflammatory potentials associated with prostate cancer. APMIS 128:80-91. https:// doi.org/10.1111/apm.13021.

9. Yu Y, Champer J, Agak GW, Kao S, Modlin RL, Kim J. 2016. Different Propionibacterium acnes phylotypes induce distinct immune responses and express unique surface and secreted proteomes. J Invest Dermatol 136: 2221-2228. https://doi.org/10.1016/j.jid.2016.06.615.

10. Cohen RJ, Shannon BA, McNeal JE, Shannon T, Garrett KL. 2005. Propionibacterium acnes associated with inflammation in radical prostatectomy specimens: a possible link to cancer evolution? J Urol 173:1969-1974. https://doi.org/10.1097/01.ju.0000158161.15277.78.

11. Fassi Fehri L, Mak TN, Laube B, Brinkmann V, Ogilvie LA, Mollenkopf H, Lein M, Schmidt T, Meyer TF, Brüggemann H. 2011. Prevalence of Propionibacterium acnes in diseased prostates and its inflammatory and transforming activity on prostate epithelial cells. Int J Med Microbiol 301:69-78. https://doi.org/10.1016/j .jjmm.2010.08.014

12. Negi M, Takemura T, Guzman J, Uchida K, Furukawa A, Suzuki Y, lida T, Ishige I, Minami J, Yamada T, Kawachi H, Costabel U, Eishi Y. 2012. Localization of Propionibacterium acnes in granulomas supports a possible etiologic link between sarcoidosis and the bacterium. Mod Pathol 25: 1284-1297. https://doi.org/10.1038/modpathol.2012.80.

13. Bae $Y$, Ito T, lida T, Uchida K, Sekine M, Nakajima $Y$, Kumagai J, Yokoyama T, Kawachi H, Akashi T, Eishi Y. 2014. Intracellular Propionibacterium acnes infection in glandular epithelium and stromal macrophages of the prostate with or without cancer. PLoS One 9:e90324. https://doi.org/10.1371/journal.pone.0090324.

14. Davidsson S, Mölling P, Rider JR, Unemo M, Karlsson MG, Carlsson J, Andersson SO, Elgh F, Söderquist B, Andrén O. 2016. Erratum to: Frequency and typing of Propionibacterium acnes in prostate tissue obtained from men with and without prostate cancer. Infect Agents Cancer 11:36. https://doi.org/10.1186/s13027-016-0084-7.

15. Solinas G, Germano G, Mantovani A, Allavena P. 2009. Tumor-associated macrophages (TAM) as major players of the cancer-related inflammation. J Leukoc Biol 86:1065-1073. https://doi.org/10.1189/jlb.0609385.

16. Locati M, Curtale G, Mantovani A. 2020. Diversity, mechanisms, and significance of macrophage plasticity. Annu Rev Pathol 15:123-147. https://doi .org/10.1146/annurev-pathmechdis-012418-012718.

17. Chen G, Liang YX, Zhu JG, Fu X, Chen YF, Mo RJ, Zhou L, Fu H, Bi XC, He HC, Yang SB, Wu YD, Jiang FN, Zhong WD. 2014. CC chemokine ligand 18 correlates with malignant progression of prostate cancer. Biomed Res Int 2014:1-10. https://doi.org/10.1155/2014/230183.

18. Gerashchenko GV, Grygoruk OV, Kononenko OA, Gryzodub OP, Stakhovsky EO, Kashuba VI. 2018. Expression pattern of genes associated 
with tumor microenvironment in prostate cancer. Exp Oncol 40:315-322. https://doi.org/10.31768/2312-8852.2018.40(4):315-322.

19. Lu D, Ni Z, Liu X, Feng S, Dong X, Shi X, Zhai J, Mai S, Jiang J, Wang Z, Wu H, Cai K. 2019. Beyond T cells: understanding the role of PD-1/PD-L1 in tumor-associated macrophages. J Immunol Res 2019:1919082. https://doi .org/10.1155/2019/1919082.

20. Dannenmann SR, Thielicke J, Stöckli M, Matter C, von Boehmer L, Cecconi V, Hermanns T, Hefermehl L, Schraml P, Moch H, Knuth A, van den Broek M. 2013. Tumor-associated macrophages subvert T-cell function and correlate with reduced survival in clear cell renal cell carcinoma. Oncoimmunology 2:e23562. https://doi.org/10.4161/onci.23562.

21. Waniczek D, Lorenc Z, Śnietura M, Wesecki M, Kopec A, Muc-Wierzgoń M. 2017. Tumor-associated macrophages and regulatory $T$ cells infiltration and the clinical outcome in colorectal cancer. Arch Immunol Ther Exp (Warsz) 65:445-454. https://doi.org/10.1007/s00005-017-0463-9.

22. Lundholm $M$, Hägglöf $C$, Wikberg $M L$, Stattin $P$, Egevad $L$, Bergh $A$, Wikström P, Palmqvist R, Edin S. 2015. Secreted factors from colorectal and prostate cancer cells skew the immune response in opposite directions. Sci Rep 5:15651. https://doi.org/10.1038/srep15651.

23. Idorn M, Køllgaard T, Kongsted P, Sengeløv L, Thor Straten P. 2014. Correlation between frequencies of blood monocytic myeloid-derived suppressor cells, regulatory T cells and negative prognostic markers in patients with castrationresistant metastatic prostate cancer. Cancer Immunol Immunother 63: 1177-1187. https://doi.org/10.1007/s00262-014-1591-2.

24. Davidsson S, Andren O, Ohlson AL, Carlsson J, Andersson SO, Giunchi F, Rider JR, Fiorentino M. 2018. FOXP3 ${ }^{+}$regulatory T cells in normal prostate tissue, postatrophic hyperplasia, prostatic intraepithelial neoplasia, and tumor histological lesions in men with and without prostate cancer. Prostate 78:40-47. https://doi.org/10.1002/pros.23442.

25. Erlandsson A, Carlsson J, Lundholm M, Fält A, Andersson SO, Andrén O, Davidsson S. 2019. M2 macrophages and regulatory T cells in lethal prostate cancer. Prostate 79:363-369. https://doi.org/10.1002/pros.23742.

26. Sfanos KS, Isaacs WB, De Marzo AM. 2013. Infections and inflammation in prostate cancer. Am J Clin Exp Urol 1:3-11.

27. Jubel JM, Barbati ZR, Burger C, Wirtz DC, Schildberg FA. 2020. The role of PD-1 in acute and chronic infection. Front Immunol 11:487. https://doi .org/10.3389/fimmu.2020.00487.

28. Curiel TJ, Coukos G, Zou L, Alvarez X, Cheng P, Mottram P, Evdemon-Hogan M, Conejo-Garcia JR, Zhang L, Burow M, Zhu Y, Wei S, Kryczek I, Daniel B, Gordon A, Myers L, Lackner A, Disis ML, Knutson KL, Chen L, Zou W. 2004. Specific recruitment of regulatory $T$ cells in ovarian carcinoma fosters immune privilege and predicts reduced survival. Nat Med 10:942-949. https://doi.org/ 10.1038/nm1093.

29. Cao Q, Wang Y, Zheng D, Sun Y, Wang Y, Lee VW, Zheng G, Tan TK, Ince J, Alexander SI, Harris DC. 2010. IL-10/TGF-beta-modified macrophages induce regulatory $T$ cells and protect against adriamycin nephrosis. J Am Soc Nephrol 21:933-942. https://doi.org/10.1681/ASN.2009060592.

30. Cai J, Qi Q, Qian X, Han J, Zhu X, Zhang Q, Xia R. 2019. The role of PD-1/PD-L1 axis and macrophage in the progression and treatment of cancer. J Cancer Res Clin Oncol 145:1377-1385. https://doi.org/10.1007/s00432-019-02879-2.

31. Li Q, Wu W, Gong D, Shang R, Wang J, Yu H. 2021. Propionibacterium acnes overabundance in gastric cancer promote $\mathrm{M} 2$ polarization of macrophages via a TLR4/PI3K/Akt signaling. Gastric Cancer 24:1242-1253. https://doi.org/10 .1007/s10120-021-01202-8.

32. Hwang SA, Ali Y, Fedotova E, Hunter RL, Brown RE. 2020. Morphoproteomics identifies the foamy alveolar macrophage as an M2 phenotype with PD-L1 expression in the early lesion of post-primary tuberculosis: implications for host immune surveillance and therapy. Ann Clin Lab Sci 50:429-438.

33. Alexeyev OA, Marklund I, Shannon B, Golovleva I, Olsson J, Andersson C, Eriksson I, Cohen R, Elgh F. 2007. Direct visualization of Propionibacterium acnes in prostate tissue by multicolor fluorescent in situ hybridization assay. $\mathrm{J}$ Clin Microbiol 45:3721-3728. https://doi.org/10.1128/JCM.01543-07.

34. Zhu Q, Wu X, Wu Y, Wang X. 2016. Interaction between Treg cells and tumorassociated macrophages in the tumor microenvironment of epithelial ovarian cancer. Oncol Rep 36:3472-3478. https://doi.org/10.3892/or.2016.5136.

35. Ferrara G, Bleck B, Richeldi L, Reibman J, Fabbri LM, Rom WN, Condos R. 2008. Mycobacterium tuberculosis induces CCL18 expression in human macrophages. Scand J Immunol 68:668-674. https://doi.org/10.1111/j .1365-3083.2008.02182.x.

36. Gandini S, Massi D, Mandalà M. 2016. PD-L1 expression in cancer patients receiving anti PD-1/PD-L1 antibodies: a systematic review and meta-analysis. Crit Rev Oncol Hematol 100:88-98. https://doi.org/10.1016/j.critrevonc.2016 .02 .001 .

37. Maruyama T, Kono K, Izawa S, Mizukami Y, Kawaguchi Y, Mimura K, Watanabe M, Fujii H. 2010. CCL17 and CCL22 chemokines within tumor microenvironment are related to infiltration of regulatory $T$ cells in esophageal squamous cell carcinoma. Dis Esophagus 23:no-429. https://doi.org/10.1111/j.1442-2050 .2009.01029.x.

38. Korbecki J, Kojder K, Simińska D, Bohatyrewicz R, Gutowska I, Chlubek D, Baranowska-Bosiacka I. 2020. CC chemokines in a tumor: a review of pro-cancer and anti-cancer properties of the ligands of receptors CCR1, CCR2, CCR3, and CCR4. Int J Mol Sci 21:8412. https://doi.org/10.3390/ijms21218412.

39. Riezu-Boj J, Larrea E, Aldabe R, Guembe L, Casares N, Galeano E, Echeverria I, Sarobe P, Herrero I, Sangro B, Prieto J, Lasarte JJ. 2011. Hepatitis C virus induces the expression of CCL17 and CCL22 chemokines that attract regulatory $T$ cells to the site of infection. J Hepatol 54:422-431. https://doi.org/10.1016/j .jhep.2010.07.014.

40. Hatam LJ, Devoti JA, Rosenthal DW, Lam F, Abramson AL, Steinberg BM, Bonagura VR. 2012. Immune suppression in premalignant respiratory papillomas: enriched functional CD4+Foxp3 + regulatory T cells and PD1/PD-L1/L2 expression. Clin Cancer Res 18:1925-1935. https://doi.org/10 .1158/1078-0432.CCR-11-2941.

41. Korbecki J, Grochans S, Gutowska I, Barczak K, Baranowska-Bosiacka I. 2020. CC chemokines in a tumor: a review of pro-cancer and anti-cancer properties of receptors CCR5, CCR6, CCR7, CCR8, CCR9, and CCR10 ligands. Int J Mol Sci 21:7619. https://doi.org/10.3390/ijms21207619.

42. Sun Z, Du C, Xu P, Miao C. 2019. Surgical trauma-induced CCL18 promotes recruitment of regulatory $T$ cells and colon cancer progression. J Cell Physiol 234:4608-4616. https://doi.org/10.1002/jcp.27245.

43. Hofman VJ, Moreilhon C, Brest PD, Lassalle S, Le Brigand K, Sicard D, Raymond J, Lamarque D, Hébuterne XA, Mari B, Barbry PJ, Hofman PM. 2007. Gene expression profiling in human gastric mucosa infected with Helicobacter pylori. Mod Pathol 20:974-989. https://doi.org/10.1038/ modpathol.3800930.

44. Huang D, Song SJ, Wu ZZ, Wu W, Cui XY, Chen JN, Zeng MS, Su SC. 2017. Epstein-Barr virus-induced VEGF and GM-CSF drive nasopharyngeal carcinoma metastasis via recruitment and activation of macrophages. Cancer Res 77:3591-3604. https://doi.org/10.1158/0008-5472.CAN-16-2706.

45. Livak KJ, Schmittgen TD. 2001. Analysis of relative gene expression data using real-time quantitative PCR and the $2^{-\Delta \Delta C T}$ method. Methods 25: 402-408. https://doi.org/10.1006/meth.2001.1262. 